\title{
Atypical lymphocytes in malaria mimicking dengue infection in Thailand
}

This article was published in the following Dove Press journal:

Research and Reports in Tropical Medicine

27 September 2010

Number of times this article has been viewed

\section{Polrat Wilairatana'}

Noppadon Tangpukdee'

Sant Muangnoicharoen'

Srivicha Krudsood ${ }^{2}$

Shigeyuki Kano ${ }^{3}$

'Department of Clinical Tropical Medicine, ${ }^{2}$ Department of Tropical Hygiene, Faculty of Tropical Medicine, Mahidol University, Bangkok, Thailand; ${ }^{3}$ Department of Tropical Medicine and Malaria, Research Institute, National Center for Global Health and

Medicine, Tokyo, Japan
Correspondence: Polrat Wilairatana Department of Clinical Tropical Medicine, Faculty of Tropical Medicine, Mahidol University, Rajvithi Road, Rajthevi, Bangkok 10400, Thailand

Tel +6623549159

Fax +662 3549158

Email tmpwl@mahidol.ac.th
Abstract: Patients with uncomplicated falciparum or vivax malaria usually present with acute febrile illness and thrombocytopenia similar to dengue infection. We retrospectively studied atypical lymphocytes (AL) and atypical lymphocytosis (ALO, defined as AL > 5\% of total white blood cells) in 1310 uncomplicated malaria patients. In 718 falciparum malaria patients, $\mathrm{AL}$ and ALO on day 0 were found in $53.2 \%$ and $5.7 \%$ of the patients, respectively, with median AL on admission of $1 \%$ (range $0 \%-10 \%$ ), whereas in 592 vivax malaria patients, $\mathrm{AL}$ and ALO on day 0 were found in $55.4 \%$ and $9.5 \%$ of the patients, respectively, with median AL on admission of $1 \%$ (range $0 \%-14 \%$ ). After antimalarial treatment, AL and ALO declined in both falciparum and vivax malaria. However, AL and ALO remained in falciparum malaria on days 7, 14, and 21, whereas AL and ALO remained in vivax malaria on days 7, 14, 21, and 28. In both falciparum and vivax malaria patients, there was a positive correlation between AL and total lymphocytes, but a negative correlation between AL and highest fever on admission, white blood cells, and neutrophils, eosinophils, and platelets $(P<0.05)$. In conclusion, AL or ALO may be found in uncomplicated falciparum and vivax malaria mimicking dengue infection. In tropical countries where both dengue and malaria are endemic, presence of AL or ALO in any acute febrile patients with thrombocytopenia (similar to the findings in dengue) malaria could not be excluded. Particularly if the patients have risk of malaria infection, confirmative microscopic examination for malaria should be carried out.

Keywords: malaria, dengue, atypical lymphocytes

\section{Introduction}

Atypical lymphocytes (AL) reactive or variant lymphocytes or transformed lymphocytes or virocytes is a nonmalignant leukocyte seen in the peripheral blood. These are reactive lymphocytes of lymphoid origin and are produced in a variety of disorders usually associated with many viral infections, eg, dengue, infectious mononucleosis. ${ }^{1}$ AL generally shows the different stages of immune responsiveness of $\mathrm{B}$ and $\mathrm{T}$ lymphocytes in the peripheral blood and immune system. AL also associates with a variety of nonviral infections, eg, tuberculosis, mycoplasma pneumonia.

Dengue infection is endemic in tropical countries. Thailand is one of the endemic countries where dengue infection accounts for $5.7 \%-10.7 \%$ of total acute febrile illness cases. ${ }^{2,3}$ Febrile illness in dengue infection has nonspecific symptoms, and in complete blood count, dengue infection usually shows AL (normal range $0 \%-5 \%$ of total white blood cells (WBC) in blood) or atypical lymphocytosis (ALO) (with AL > 5\% of total WBC). ${ }^{4-7}$ Oliveira et al (2009) have shown that the main hematological findings in dengue included leucopenia (68.3\%), thrombocytopenia (66.5\%), lymphocytopenia 
(67.2\%), and AL (67\%). In dengue endemic area, acute febrile patients presenting with thrombocytopenia and AL were frequently diagnosed as having dengue infection before definitive diagnosis by serology or antigen were confirmed. ${ }^{7}$ At present, supportive and symptomatic treatment, eg, hydration or platelet transfusion in those patients with systemic bleeding, is the main treatment for dengue infection.

Many malaria patients can present with nonspecific symptoms and similar complete blood count findings, eg, thrombocytopenia, which is the same picture as in dengue infection. We believe that the presence of AL may also be an important laboratory clue for diagnosis of some nonviral diseases such as malaria. Unfortunately, complete blood count by automated cell counters (or automated machines) cannot detect malaria parasites.

Some reports have shown that AL can also be found in falciparum and vivax malaria infections which range from $0 \%$ to $100 \% .^{9-14}$ These can cause unaware clinicians to misdiagnose malaria as dengue infection, particularly in a dengue-endemic area, patients present with nonspecific symptoms and a complete blood count show thrombocytopenia with AL or ALO and a microscopic blood examination for malaria has not been carried out. However, there was no study of the prevalence of AL in Thai malaria patients or of dynamic change of AL in malaria after antimalarial treatment.

As a consequence, similar complete blood counts and not conducting microscopic blood examination to look for malaria parasites, which results in delayed treatment of patients with uncomplicated malaria, can lead to severe malaria or even death. In Thailand, many severe malaria patients were previously misdiagnosed and treated for dengue infection. We believe that AL, ALO, and their correlation can be useful in diagnosis and treatment of malaria and dengue infection.

The objectives of the study were to (i) find the prevalence of AL and ALO in uncomplicated Plasmodium falciparum and Plasmodium vivax malaria, (ii) investigate dynamic changes in AL, and (iii) find the correlation of AL with other clinical and laboratory parameters.

\section{Material and methods}

\section{Study site and recruitment procedure}

The medical records of 1310 adult inpatients with uncomplicated $P$. falciparum or $P$. vivax malaria were reviewed retrospectively. Each patient (i) had been admitted to the Hospital for Tropical Diseases, Faculty of Tropical Medicine, Mahidol University, Bangkok, Thailand, for at least 28 days, for the treatment of acute uncomplicated $P$. falciparum or $P$. vivax malaria between January 2000 and December 2006, either males or females, body weight $\geq 35 \mathrm{~kg}$, age $\geq 15$ years; (ii) was positive for asexual forms of either $P$. falciparum or $P$. vivax parasites on admission, before treatment, with microscopic confirmation; (iii) was treated with artesunate plus mefloquine for uncomplicated $P$. falciparum or artesunate followed by primaquine for $P$. vivax malaria infection; (iv) had no history of antimalarial therapy preadmission for 3 months; (v) had $P$. falciparum or $P$. vivax cleared from peripheral blood with therapy and had no parasite reappearance during 28 days of admission in the hospital. There were 194 P. falciparum malaria cases in this study having dengue serologic tests. ${ }^{15}$ Exclusion criteria included patients (i) who had been given blood transfusion during the study period; (ii) with evidence of Plasmodium ovale, Plasmodium malariae, or mixed malaria infection; (iii) with evidence of severe malaria during treatment for uncomplicated malaria; ${ }^{13}$ (iv) with clinical evidence of severe malnutrition or clinically significant disorder; (v) who received treatment with antibiotics, corticosteroids, platelet-lowering agents, or immunosuppressive drugs; (vi) and with lactation or pregnancy. Of 1310 malaria patients matching the inclusion criteria, 718 had uncomplicated $P$. falciparum malaria and 592 uncomplicated $P$. vivax infection. The study was approved by the Ethics Committee, Faculty of Tropical Medicine, Mahidol University, Thailand.

\section{Baseline and follow-up studies}

Age, gender, history of malaria in the past year, initial highest fever and duration of fever preadmission, and initial parasite count were recorded. Baseline and follow-up laboratory data, eg, complete blood count, were determined by automated cell counter (Advia 120 Hematology System; Siemens Medical Solutions Diagnostics; New York, NY; commercial reagent by Roche Diagnostics, Bangkok, Thailand). WBC counts including AL were manually checked again microscopically. Thick and thin blood films for malaria parasite determinations were prepared from fingerprick blood samples and stained with Giemsa. Peripheral blood malaria parasite count of asexual P. falciparum or $P$. vivax was routinely calculated by counting the number of asexual forms per $200 \mathrm{WBC}$ on thick smears and multiplying by WBC count or by counting the number of asexual forms per 1000 erythrocytes on thin smears and multiplying by the red blood cell count. Parasite density counts were conducted every $6 \mathrm{~h}$ until disappearance of malaria parasite from peripheral blood, and then blood films were conducted once daily until day 28 of admission. Blood smears were considered negative if no parasites were seen in 200 oil-immersion microscopic fields. Parasite clearance time 
(PCT) was defined as the period from the start of treatment until the first negative blood film.

Throughout this time, WBC counts were collected for these patients every 7 days during the study period, starting from the first day of examination and then on days $7,14,21$, and 28. ALO was considered when AL was found $>5 \%$ of total WBC in circulation.

Physical examination was performed by the research physician every day, and vital signs, including blood pressure $(\mathrm{mmHg})$, heart rate (beats/min), respiratory rate (breaths/min), and body temperature $\left({ }^{\circ} \mathrm{C}\right)$, were measured every $4 \mathrm{~h}$ by the research nurse until the patients were discharged from the hospital. Fever clearance time (FCT) was taken as the period from the start of treatment until oral temperature decreased to $37.5^{\circ} \mathrm{C}$ and remained below this temperature for the next $48 \mathrm{~h}$.

\section{Treatment}

Upon admission, the patients received the following treatments:

1. P.falciparum infection: Oral artesunate $200 \mathrm{mg} /$ day single dose for 3 days, together with mefloquine $8 \mathrm{mg} / \mathrm{kg} /$ day for 3 days.

2. P. vivax infection: Oral artesunate $200 \mathrm{mg}$ single dose on day 0 followed by single daily doses of $100 \mathrm{mg}$ for the next 4 days, then primaquine $(0.6 \mathrm{mg} / \mathrm{kg})$ was administered orally once daily for 14 days.

\section{Statistical analysis}

All $P$ values reported were from two-tailed test, and statistical significance was set at 0.05 . The Kolmogorov-Smirnov test was used to test for normality, but the data distributions generally did not exhibit normality. Therefore, the data were expressed as medians (with ranges) and number of observations with percentage (\%). Three statistical tests were performed: (i) the Chi-square test was used to test for any association between qualitative variables, (ii) the Mann-Whitney $U$ test was used to test for differences between quantitative variables and the relationships among WBC counts, parasite densities, age, highest temperature during the first $72 \mathrm{~h}$ of admission, and (iii) reported days of fever preadmission were analyzed using Spearman's rank correlation coefficient.

\section{Results}

\section{Patient characteristics}

Our sample consisted of a total of 1310 patients, 718 of whom were infected with $P$. falciparum and 592 with $P$. vivax. Around 5\% were lost to follow-up by withdrawing consent or due to social reasons unrelated to adverse effects. The data obtained for such patients, before loss to follow-up, were included in analysis.

Patient characteristics are shown in Table 1. There was a statistically significant difference $(P=0.017)$ between the age distributions of the two groups. Nevertheless, the median age for the $P$. falciparum group was only 2 years more than the $P$. vivax group; the oldest patient in the $P$. falciparum group was 65 years and the one in the $P$. vivax group was 61 . Clinically or biologically, these differences were unlikely to be significant. There were statistically significant differences $(P<0.001)$ between gender, malarial parasite density, and highest temperature on admission of the two patient groups.

\section{Atypical lymphocytes and atypical lymphocytosis Falciparum malaria}

In 718 falciparum malaria patients, AL was found in 381 cases (53.1\%) on day 0 of admission (Table 2). ALO was found in 51 patients $(7.1 \%)$ out of 718 patients. Out of 381 patients with AL, the median AL on days 0, 7, 14, 21, and 28 of admission were $3 \%, 2 \%, 2 \%, 2 \%$, and $1 \%$, respectively, with the range being 1\%-17\%. ALO was also found on days 7,14 , and 21 in $16(2.2 \%), 10(1.4 \%)$, and $2(0.8 \%)$ of falciparum malaria patients, respectively; however, ALO was not found on day 28.

Figure 1 showed dynamic change of AL in all falciparum malaria patients. AL and ALO declined after antimalarial treatment. Platelets increased in number after treatment, and all patients had normal platelet counts by day 7 of treatment. Hematocrit showed slight increase from $37 \%$ on day 0 to $39 \%$ and $40 \%$ on day 21 and 28 of admission, respectively. FCT was $24 \mathrm{~h}$.

\section{Vivax malaria}

In 592 vivax malaria patients, AL was found in 328 cases (55.4\%) on day 0 of admission (Table 3 ). ALO was found in 56 patients $(9.5 \%)$ out of 592 patients. Out of 592 patients with AL, the median AL on days $0,7,14,21$, and 28 of admission were $3 \%, 2 \%, 2 \%, 2 \%$, and $1 \%$, respectively, with the range being $1 \%-14 \%$. ALO was also found on days 7,14 , 21 , and 28 in $13(2.2 \%), 4(0.7 \%), 4(0.7 \%)$, and $2(0.3 \%)$ of vivax malaria patients, respectively.

Figure 2 showed dynamic change of AL in all vivax malaria patients. AL and ALO declined after antimalarial treatment. Platelets increased in number after treatment, and all patients had normal platelet counts by day 7 of treatment. Hematocrit showed gradual increase from $37 \%$ on day 0 to $38 \%$ and $40 \%$ on day 21 and 28 of admission, respectively. FCT was $12 \mathrm{~h}$. 
Table I Baseline patient characteristics

\begin{tabular}{|c|c|c|c|}
\hline Characteristic & P. falciparum $(N=718)$ & P. vivax $(\mathrm{N}=592)$ & $P$ values \\
\hline \multicolumn{4}{|l|}{ Sex } \\
\hline (Male (\%)/Female (\%)) & $544(76 \%) / 174(24 \%)$ & $38|(64 \%) / 2| I(36 \%)$ & $<0.001$ \\
\hline \multicolumn{4}{|l|}{ Age $(y r)$} \\
\hline Median (range) & $24(15-65)$ & $22(|5-6|)$ & 0.017 \\
\hline \multicolumn{4}{|l|}{$\begin{array}{l}\text { Malaria parasite density on day } 0 \\
\text { (parasites } / \mu \mathrm{L})\end{array}$} \\
\hline Median (range) & II,840 (I30-179,040) & $9970(122-88,480)$ & $<0.001$ \\
\hline \multirow{2}{*}{\multicolumn{4}{|c|}{$\begin{array}{l}\text { Reported days of fever before } \\
\text { admission (days) }\end{array}$}} \\
\hline & & & \\
\hline Median (range) & $4(1-7)$ & $4(1-5)$ & 0.858 \\
\hline Highest temperature during & & 37.9 & \\
\hline \multicolumn{4}{|l|}{$\begin{array}{l}\text { Highest temperature during } \\
\text { admission }\left({ }^{\circ} \mathrm{C}\right)\end{array}$} \\
\hline Median (range) & $38.3(37.9-41.2)$ & $(37.6-42.3)$ & $<0.001$ \\
\hline \multicolumn{4}{|l|}{$\mathrm{CBC}$ profiles } \\
\hline \multicolumn{4}{|l|}{ Median (range) } \\
\hline Hemoglobin (g/dL) (range) & $12.2(5.2-17.8)$ & $12.1(3.1-17.2)$ & 0.906 \\
\hline Hematocrit (\%) (range) & $37(17-53)$ & $37(10-52)$ & 0.317 \\
\hline WBC $\left(\times 10^{3} / \mu \mathrm{L}\right)($ range $)$ & $5.1(2.2-13.1)$ & $5.7(91.9-13.50)$ & $<0.001$ \\
\hline$\%$ of neutrophils (range) & $63(19-88)$ & $64(29-88)$ & 0.752 \\
\hline$\%$ of eosinophils (range) & $2(0-22)$ & I (0-22) & 0.094 \\
\hline$\%$ of lymphocytes (range) & $21(95-58)$ & $21(4-55)$ & 0.761 \\
\hline$\%$ of $\mathrm{AL}$ (range) & $\mid(0-17)$ & I (0-14) & 0.436 \\
\hline Platelet count $\left(\times 10^{3} / \mu \mathrm{L}\right)$ (range) & $56(15-347)$ & $79(12-294)$ & 0.094 \\
\hline
\end{tabular}

Abbreviations: $\mathrm{CBC}$, complete blood count; WBC, white blood cells; AL, atypical lymphocytes.

In both falciparum and vivax malaria patients, there was positive correlation between AL and total lymphocytes but negative correlation between $\mathrm{AL}$ and highest fever on admission, WBC, and neutrophils, eosinophils, and platelets $(P<0.05)$.

\section{Discussion}

Dengue infection was the third cause, after leptospirosis and scrub typhus, of acute undifferentiated, febrile illness in the absence of an obvious focus of infection in rural Thailand. ${ }^{3}$ Concurrent dengue and malaria infection can be found and varies in different regions. Although most malaria patients in this study had not undergone serological tests for dengue infection, only 194 (15\%) of all 1310 patients in this study were serologically tested for dengue, and no positive serological diagnosis of dengue was demonstrated as co-infection in the malaria patients. ${ }^{15}$ Co-infection might be absent or uncommon in our AL study. Failure to send the serum of all the

Table $2 \mathrm{AL}$ found in falciparum malaria patients $(n=718)$

\begin{tabular}{|c|c|c|c|c|c|}
\hline$\%$ of $A L$ & Day 0 n (\%) & Day 7 n (\%) & Day I4 n (\%) & Day 2 I n (\%) & Day 28 n (\%) \\
\hline 0 & $337(46.8)$ & $37 \mid(5 । .7)$ & $546(76)$ & $638(88.8)$ & $637(88.6)$ \\
\hline I & $90(12.5)$ & $95(13.3)$ & $62(8.6)$ & $36(5)$ & $49(6.8)$ \\
\hline 2 & $90(12.5)$ & $106(14.7)$ & $57(8.0)$ & $24(3.4)$ & $24(3.2)$ \\
\hline 3 & 84 (11.7) & $67(9.3)$ & $19(2.7)$ & $14(1.9)$ & $4(0.6)$ \\
\hline 4 & $51(7.2)$ & $35(4.8)$ & $13(1.8)$ & ND & $2(0.3)$ \\
\hline 5 & $14(1.9)$ & $28(4.0)$ & $9(1.2)$ & $4(0.6)$ & $2(0.3)$ \\
\hline 6 & 24 (1.9) & $4(0.6)$ & $6(0.9)$ & ND & ND \\
\hline 7 & $15(2.2)$ & $4(0.6)$ & ND & $2(0.3)$ & ND \\
\hline 8 & $6(0.8)$ & $8(1.1)$ & $2(0.3)$ & ND & ND \\
\hline 9 & ND & ND & $2(0.3)$ & ND & ND \\
\hline 10 & $6(0.8)$ & ND & ND & ND & ND \\
\hline 11 & ND & ND & ND & ND & ND \\
\hline 14 & ND & ND & ND & ND & ND \\
\hline 17 & ND & ND & $2(0.3)$ & ND & ND \\
\hline
\end{tabular}

Note: Numbers of patients with ALO are shown in bold.

Abbreviation: ND, not detectable. 


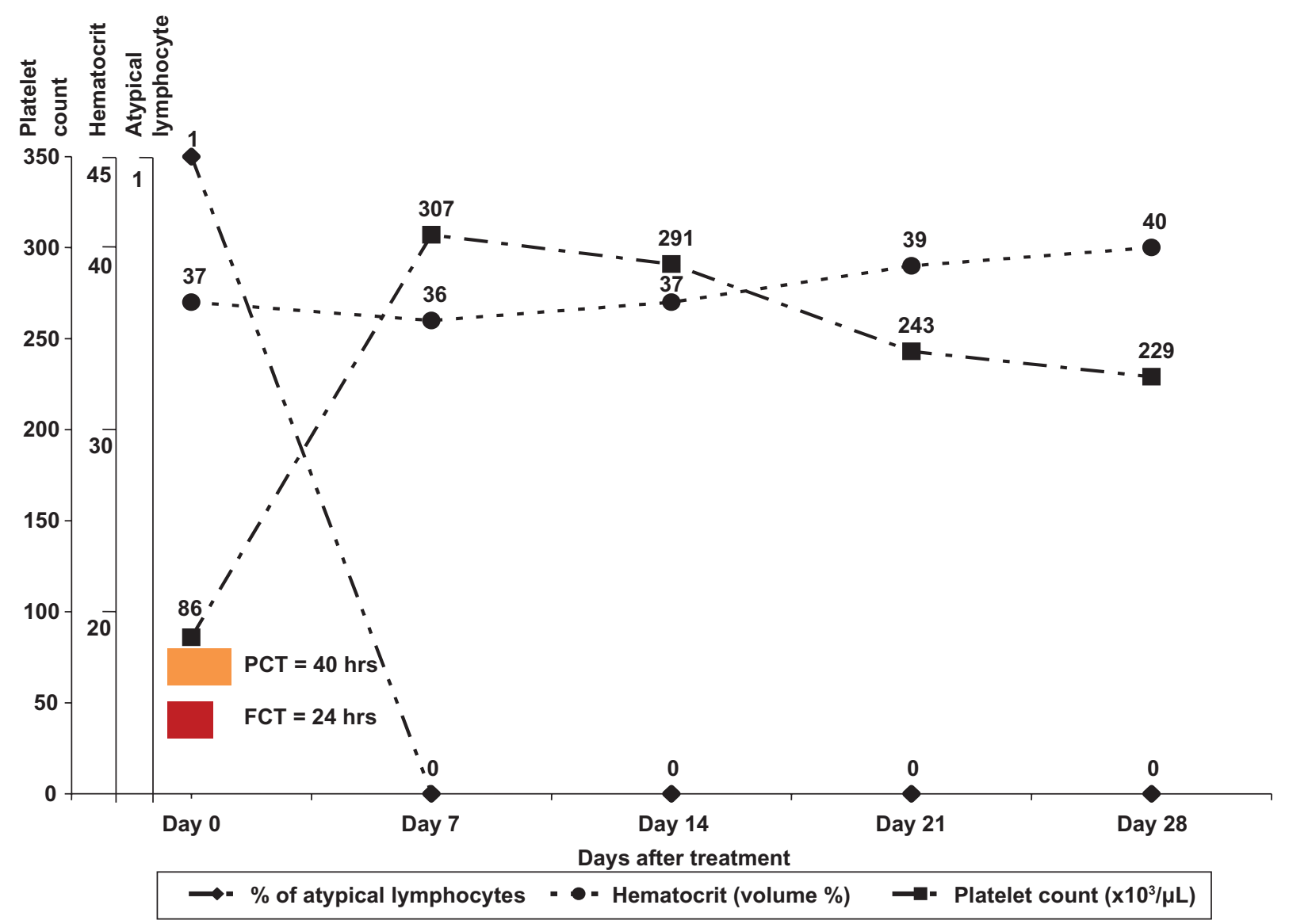

Figure I Median of percentage of atypical lymphocytes, hematocrit, and platelet count in patients infected with $P$. falciparum malaria. Abbreviations: PCT, parasite clearance time; FCT, fever clearance time.

patients for dengue serologic tests was the flaw of the study. However, 194 malaria patients were a rather big sample. If malaria-dengue co-infection was common, serological test for dengue infection should be found positive at least in one or some of the 194 patients. However, dengue infection was common in some countries, eg, Pakistan, showing dual dengue and malaria in $23.2 \%$ of the acute febrile patients. ${ }^{16}$ One possibility why malaria-dengue co-infection is uncommon in Thailand where malaria and dengue are endemic is because Anopheles mosquitoes, vectors for malaria, breed

Table $3 \mathrm{AL}$ found in vivax malaria patients $(n=592)$

\begin{tabular}{|c|c|c|c|c|c|}
\hline$\%$ of $A L$ & Day 0 n (\%) & Day 7 n (\%) & Day I 4 n (\%) & Day 2 I n (\%) & Day 28 n (\%) \\
\hline 0 & $264(44.6)$ & $381(64.3)$ & $480(8 \mathrm{I})$ & $508(85.8)$ & $588(94.3)$ \\
\hline I & 75 (12.7) & $86(14.7)$ & $52(8.7)$ & $37(6.4)$ & $14(2.3)$ \\
\hline 2 & $88(14.9)$ & $63(10.8)$ & $30(5.2)$ & $20(3.3)$ & $12(2)$ \\
\hline 3 & $50(8.4)$ & $27(4.6)$ & $9(1.6)$ & II (I.8) & $4(0.7)$ \\
\hline 4 & $30(5.1)$ & $14(2.3)$ & II (I.9) & $5(0.9)$ & ND \\
\hline 5 & $33(5.6)$ & $8(1.3)$ & $6(1.1)$ & $7(1.2)$ & $2(0.3)$ \\
\hline 6 & $17(2.8)$ & $6(1)$ & $2(0.3)$ & ND & ND \\
\hline 7 & $20(3.3)$ & $3(0.5)$ & ND & $2(0.3)$ & ND \\
\hline 8 & $5(0.8)$ & ND & ND & ND & ND \\
\hline 9 & $5(90.8)$ & ND & ND & ND & ND \\
\hline 10 & $5(0.8)$ & $2(0.3)$ & ND & ND & ND \\
\hline II & $2(0.3)$ & $2(0.3)$ & ND & $2(0.3)$ & $2(0.3)$ \\
\hline 13 & ND & ND & $2(0.3)$ & ND & ND \\
\hline 14 & $2(0.3)$ & ND & ND & ND & ND \\
\hline
\end{tabular}

Note: Numbers of patients with ALO are shown in bold. Abbreviation: ND, not detectable. 


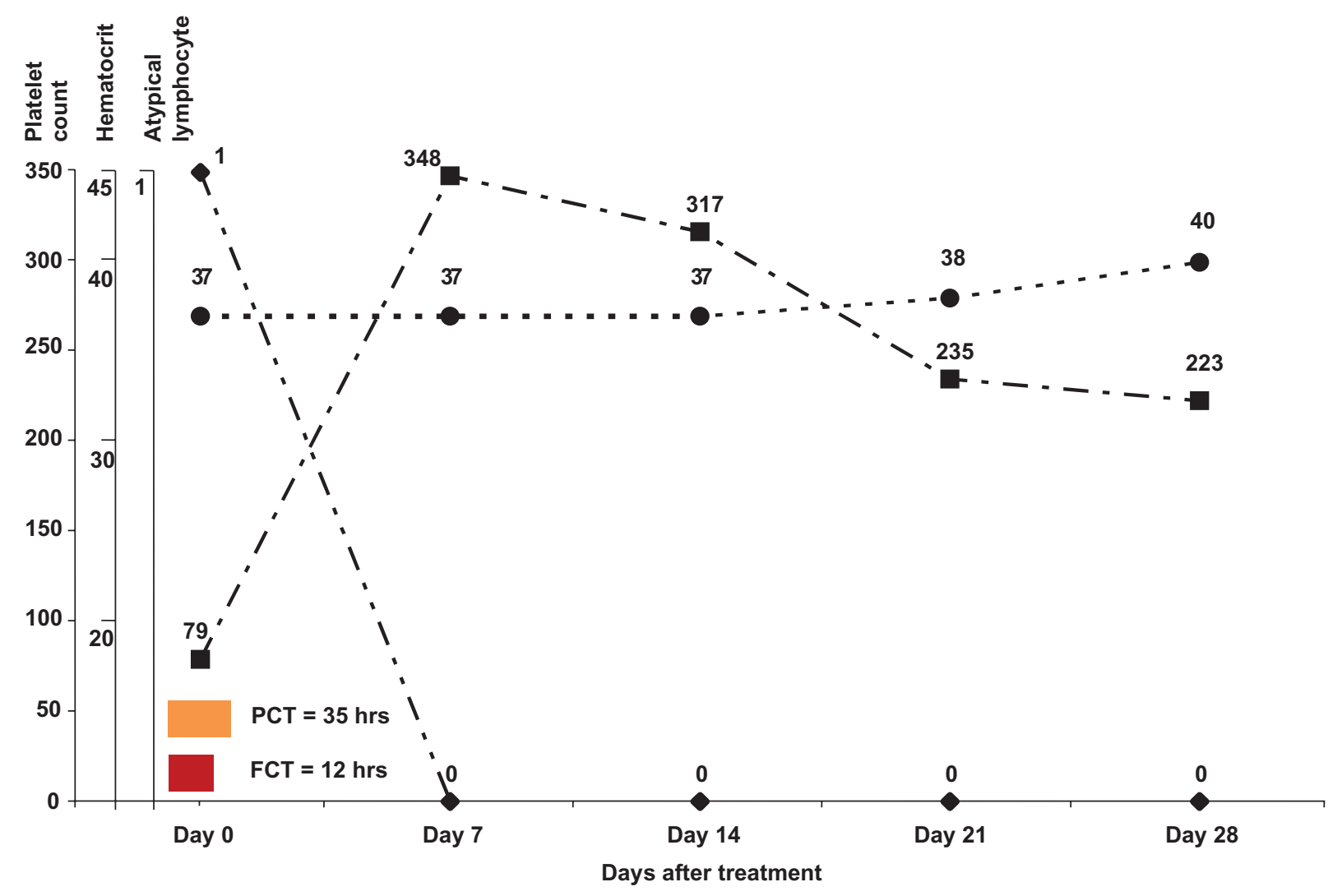

$\rightarrow \%$ of atypical lymphocytes - - Hematocrit (volume \%) $\rightarrow$ Platelet count $\left(\times 10^{3} / \mu L\right)$

Figure 2 Median of percentage of atypical lymphocytes, hematocrit, and platelet count in patients infected with $P$. vivax malaria. Abbreviations: PCT, parasite clearance time; FCT, fever clearance time.

and are found in forested areas, whereas Aedes aegypti mosquitoes, vectors for dengue, breed and are found mostly in urban areas. This different distribution in disease vectors could answer why we uncommonly found malaria-dengue co-infection.

In falciparum malaria, on day $0, \mathrm{AL}$ and ALO were found in $53.2 \%$ and $5.7 \%$ of the patients, respectively. The highest percentage of AL on day 0 was $10 \%$, which was found in only $0.8 \%$ of the patients. AL and ALO were also observed on days 7 and 14 . On day 28 , AL was found in $11.4 \%$ of the patients; however, no ALO was demonstrated. In vivax malaria, on day $0, \mathrm{AL}$ and ALO were found in $55.4 \%$ and $9.1 \%$ of the patients, respectively. The highest percentage of AL on day 0 was $14 \%$, which was found in only $0.3 \%$ of the patients. AL and ALO were also observed on days 7, 14, and 28. On day 28, AL and ALO were found in 5.7\% and $0.3 \%$ of the patients, respectively.

As shown in Figures 1 and 2, AL in both falciparum and vivax malaria tended to decline after antimalarial treatment. Platelet counts tended to increase in number after antimalarial treatment. Hematocrit gradually increased in convalescence.
The sudden rise of hematocrit or hemoconcentration (defined as $20 \%$ increase in hematocrit as the evidence of plasma leakage), typically found in dengue hemorrhagic fever, was not found in our study. ${ }^{17}$ Thisyakorn et al found that the percentage of $\mathrm{AL}$ in dengue hemorrhagic fever was $<5 \%$ 2 days before onset of subsidence of fever or shock and that there was significant rise of AL (with mean $26 \% \pm 12 \%$ ) in both shock and non-shock dengue hemorrhagic fever patients. ${ }^{5}$

This study showed that AL and ALO could be found in malaria patients up to day 28 after proper antimalarial treatment. Although many literatures studied outside Thailand showed that patients with malaria and dengue could present with similar findings, eg, acute febrile illness, complete blood count showing thrombocytopenia, and increase in $\mathrm{AL}$ and/ or ALO, many clinicians particularly in Thailand were not aware of these issues and thought that any acute febrile patient with thrombocytopenia, AL, or ALO had dengue infection. Another important issue is that the automated machine available in many hospitals used for determining the $\mathrm{CBC}$ count cannot detect malaria. Therefore, if clinicians do not think to ask the patients for their travel history in malaria endemic 
areas, or do not request for a malaria test to be done at the laboratory, nearly all malaria patients will be misdiagnosed as having dengue, resulting in delays in diagnosis and treatment of malaria, which can contribute to patients developing from uncomplicated malaria to severe malaria or even a more fatal outcome.

There was no previous documented report that antimalarial drugs used in this study can cause change in AL counts. Apart from malaria, we did not do other laboratory tests to confirm infectious and noninfectious causes which could cause AL or ALO, eg, tuberculosis, post irradiation, thyrotoxicosis, rheumatoid arthritis, myasthenia gravis, ${ }^{1}$ since there was no clue from history, and no obvious clinical manifestations that suggested those diagnoses.

\section{Conclusion}

AL could be found in patients infected with both falciparum and vivax malaria even after treatment up to 28 days after proper antimalarial treatment. AL gradually decreased after treatment, and platelet counts gradually increased in both falciparum and vivax malaria patients. Fever, thrombocytopenia, AL, or ALO do not mean only dengue infection; malaria should be in differential diagnosis of acute febrile patients present with thrombocytopenia and AL or ALO, especially in tropical countries where dengue and malaria are endemic. Early diagnosis and prompt appropriate treatment of malaria will reduce morbidity and mortality of the patients.

\section{Acknowledgment}

We thank Dove Press for printing support.

\section{Disclosure}

The authors report no conflicts of interest in this work.

\section{References}

1. Simon MW. The atypical lymphocyte. Int Pediatr. 2003;18:20-22.

2. Leelarasamee A, Chupaprawan C, Chenchittikul M, et al. Etiologies of acute undifferentiated febrile illness in Thailand. J Med Assoc Thai. 2004;87:464-472.

3. Suttinont C, Losuwanaluk K, Niwastayakul K, et al. Causes of acute, undifferentiated, febrile illness in rural Thailand: results of a prospective observational study. Ann Trop Med Parasitol. 2006;100: 363-370.

4. Suvatte V, Longsaman M. Diagnostic value of buffy coat preparation in dengue hemorrhagic fever. Southeast Asian J Trop Med Public Health. 1979;10:7-12.

5. Thisyakorn U, Nimmanniya S, Ningsanond V, et al. Atypical lymphocyte in dengue hemorrhagic fever: its value in diagnosis. Southeast Asian $J$ Trop Med Public Health. 1984;15:32-36.

6. Hoffman R, Benz, Shattil SJ, et al. Hematology: Basic Principals and Practice. 5th ed. Philadelphia, PA: Churchill Livingstone, Elsevier; 2009.

7. Oliveira EC, Pontes ER, Cunha RV, et al. Hematological abnormalities in patients with dengue. Rev Soc Bras Med Trop. 2009;42: 682-685.

8. WHO. Dengue: Guidelines for Diagnosis, Treatment, Prevention and Control. France: WHO/HTM/NTD/DEN/2009.1. 2009. p. 25-55.

9. Wells RA, Scott RM, Pavanand K, et al. Kinetics of peripheral blood leukocyte alterations in Thai children with dengue hemorrhagic fever. Infect Immun. 1980;28:428-433.

10. Cunha BA, Bonoan JT, Schlossberg D. Atypical lymphocytes in acute malaria. Arch Intern Med. 1997;157:1140-1141.

11. Yoon JH, Cho HI. Appearance characteristics of the atypical lymphocytes in the blood of the Plasmodium vivax malarial patients. Korean J Hematol. 2001;36:123-128.

12. Bashawri LAM, Mandil AA, Bahnassy AA, et al. Malaria: hematological aspects. Ann Saudi Med. 2002;22:372-377.

13. Taha K, El-Dein SZ, Indrees M, et al. Hematological changes in malaria: relation to Plasmodium species. Kuwait Med J. 2007;39:262-267.

14. World Health Organization. Guidelines for the Treatment of Malaria. 2nd ed. Geneva, Switzerland: WHO; 2010.

15. Singsilarak T, Phongtananant S, Jenjitikul M, et al. Possible acute coinfections in Thai malaria patients. Southeast Asian J Trop Med Public Health. 2006;37:1-4.

16. Abbasi A, Butt N, Sheikh QH, et al. Clinical features, diagnostic techniques and management of dual dengue and malaria infection. $J$ Coll Physicians Surg Pak. 2009;19:25-29.

17. Kallayanarooj S, Nimmannitya S. Guidelines for Dengue Hemorrhagic Fever Case Management. Bangkok, Thailand: Bangkok Medical Publisher; 2004:1-22.
Research and Reports in Tropical Medicine

\section{Publish your work in this journal}

Research and Reports in Tropical Medicine is an international, peerreviewed, open access journal publishing original research, case reports, editorials, reviews and commentaries on all areas of tropical medicine, including: Diseases and medicine in tropical regions; Entomology; Epidemiology; Health economics issues; Infectious disease; Laboratory

\section{Dovepress}

science and new technology in tropical medicine; Parasitology; Public health medicine/health care policy in tropical regions; and Microbiology. The manuscript management system is completely online and includes a very quick and fair peer-review system. Visit http://www.dovepress. com/testimonials.php to read real quotes from published authors. 